\title{
Serum Highly Sensitive Cardiac Troponin I (hs-cTnI) as a Diagnostic Biomarker of Myocardial Injury after Pediatric Cardiac Catheterization Ahmed Magdy Hegazy ${ }^{1}$, Raghda Ghonimy El-Sheikh ${ }^{2}$, Desoky Ezzat Abo Ammo ${ }^{3}$ and Amr Mohamed Zoair ${ }^{1 *}$ Departments of ${ }^{1}$ Pediatric Medicine, ${ }^{2}$ Cardiology and ${ }^{3}$ Clinical Pathology, Faculty of Medicine, Tanta University, Egypt \\ *Corresponding author: Ahmed Magdy Hegazy; Mobile: 01062196986; E-mail: heg_ahmed88@yahoo.com
}

\begin{abstract}
Background: new Highly Sensitive Cardiac Troponin I assay (as hs-cTnI) represents an important advance with added sensitivity for cardiac myocyte necrosis and could have several distinct roles in clinical practice.

Aim of the present study: it was to determine the serum level of highly sensitive cardiac troponin I (hs-cTnI) before and after pediatric cardiac catheterization (diagnostic or interventional), as a diagnostic biomarker of myocardial injury in these patients. Patients and Methods: a Prospective Cohort study was carried out during the period from January 2017 to June 2018 at Pediatric Cardiology Unit and Cardiology Department, Tanta University Hospital. It included 30 patients who were admitted and required pediatric cardiac catheterization. Doppler echo- cardiography and Estimation of serum highly sensitive cardiac troponin I (hs-cTnI): using the ELFA technique (Enzyme Linked Fluorescent Assay) was done. Results: there was highly significant increase of serum hs-cTnI levels immediately after and 12 hours after therapeutic pediatric cardiac catheterization ( $\mathrm{p}<0.001$ ), whereas in diagnostic pediatric cardiac catheterization there was no significant differences of serum hs-cTnI levels $(\mathrm{p}>0.05)$. There was significant negative correlation between serum hscTnI levels and Fractional shortening (FS) \%, and also there was significant negative correlation between serum hs-cTnI levels and ejection fraction (EF) \% (measured by echocardiography). Conclusion: serum highly sensitive cardiac troponin I (hs-cTnI) was significantly increased immediately after pediatric cardiac catheterization, with more significant increase after 12 hours, suggesting myocardial injury in these patients. Also, elevation of this diagnostic biomarker of myocardial injury was more after therapeutic than diagnostic pediatric cardiac catheterization.

Keywords: Highly Sensitive Cardiac Troponin I, Myocardial Injury, Pediatric Cardiac Catheterization
\end{abstract}

\section{INTRODUCTION}

Cardiac catheterization has long served as the "gold standard" for the anatomic and physiological assessment of patients with CHD. Real-time fluoroscopy with contrast injection coupled with rapid digital angiography has provided the high-resolution images of the heart necessary for successful surgical management of these patients ${ }^{(\mathbf{1})}$.

It has been shown that after a myocardial infarction the heart initially releases free cTnT into the plasma. Later, it releases free cTnI, tertiary cTnT-cTnI-TnC complexes, and, occasionally, some fragments of cTnT. Tertiary complexes have a short half-life, since they are rapidly broken down into free cTnT and cTnI-TnC binary complexes ${ }^{(\mathbf{1})}$.

With advances in technology, a new era in troponin assays is approaching, previous-generation troponin assays have been used as diagnostic and prognostic markers in acute coronary syndrome patients and for risk stratification to guide triage decisions and aid in treatment selection. New, high-sensitivity troponin assays represent an important advance with added sensitivity for cardiac myocyte necrosis, but there remains a need for judicious interpretation with these tests ${ }^{(3)}$.

The objective of the present study was to determine the serum level of highly sensitive cardiac troponin I (hs-cTnI) before and after pediatric cardiac catheterization (diagnostic or interventional), as a diagnostic biomarker of myocardial injury in these patients.

\section{PATIENTS AND METHODS}

This Prospective Cohort study was carried out during the period from January 2017 to June 2018 at Pediatric Cardiology Unit and Cardiology Department, Tanta University Hospital. It included 30 patients who were admitted and required pediatric cardiac catheterization. The study was approved by the Ethics Board of Tanta University and an informed written consent was taken from each participant in the study.

Inclusion criteria: Infants and children aged from 6 months to 12 years with cardiac diseases admitted for cardiac catheterization for diagnostic or interventional purposes both sexes were included.

Exclusion criteria: Infants and children with heart failure, cardiomyopathy, myocarditis, acute or chronic infection, chronic renal failure, muscle diseases, neoplastic diseases, or high troponin level before catheterization.

Written Informed consent was obtained from the parents or guardians of the child. The study was approved 
by the Ethics Committee of Faculty of Medicine, Tanta University.

Collection of data: All infants and children were subjected to:

1. Complete History Taking: Including age, sex, weight, and onset of symptoms, course of the disease and aim of cardiac catheterization.

2. Thorough Clinical Examination: Including all body systems, vital data, general examination, chest, heart, abdomen, CNS, and complete local cardiac examination.

\section{Investigations:}

- Plain chest x- ray.

- Electrocardiography: A standard 12 lead ECG recorded with the machine calibrated with paper speed at $25 \mathrm{~mm} / \mathrm{sec}$ and amplitude of stylus deflection at $1 \mathrm{mV} / \mathrm{cm}$.

- Standard transthoracic M-mode, two dimensional Doppler echocardiographic studies: performed using an ACUSONCV70 Echo Doppler machines equipped with a2.5\3.2-MHZ annular array transducer for diagnosis of type of cardiac disease and assessment of cardiac function.

- Cardiac catheterization ${ }^{(4)}$.
Estimation of serum highly sensitive cardiac troponin I (hs-cTnI): using the ELFA technique (Enzyme Linked Fluorescent Assay) ${ }^{(5)}$.

\section{Statistical analysis}

In addition to the descriptive data, statistical analysis was done using IBM SPSS STATISTC VERSION 23 PROGRAM. Data were expressed as mean $\pm \mathrm{SD}$ and analyzed using the Student's t-test, Paired (t) test and ANOVA test to assess the significance of difference in the levels between different parameters. $\mathrm{P}$ $<0.05$ was accepted as significant. Coefficient (r) of two variables was also done by using Pearson Correlation Coefficient (r) with P Value Calculation.

\section{RESULTS}

Laboratory assessments of the measured parameters are presented in the following tables and figures:

Table (1): Demographic data of the patients

\begin{tabular}{|c|c|c|c|}
\hline & No. $=30$ & & No. $=30$ \\
\hline $\begin{array}{l}\text { Age (years) } \\
\text { Mean } \pm \text { SD }\end{array}$ & $3.43 \pm 2.80$ & $\begin{array}{l}\text { Weight }(\mathbf{k g}) \\
\text { Mean } \pm \text { SD }\end{array}$ & $14.15 \pm 7.58$ \\
\hline $\begin{array}{r}\text { Age category } \\
<4 \text { years } \\
\end{array}$ & $20(66.7 \%)$ & $\begin{array}{r}\text { Weight category } \\
<15 \mathrm{~kg} \\
\end{array}$ & $21(70.0 \%)$ \\
\hline$>4$ years & $10(33.3 \%)$ & $>15 \mathrm{~kg}$ & $9(30.0 \%)$ \\
\hline Females & $12(40.0 \%)$ & $\begin{array}{l}\text { BMI category } \\
<15 \mathrm{~kg} / \mathrm{m} 2\end{array}$ & $12(40.0 \%)$ \\
\hline Males & $18(60.0 \%)$ & $>15 \mathrm{~kg} / \mathrm{m} 2$ & $18(60.0 \%)$ \\
\hline $\begin{array}{c}\text { Length }(\mathbf{c m}) \\
\text { Mean } \pm \text { SD }\end{array}$ & $93.90 \pm 21.78$ & $\begin{array}{c}\text { BMI }\left(\mathbf{k g} / \mathbf{m}^{2}\right) \\
\text { Mean } \pm \text { SD }\end{array}$ & $15.61 \pm 2.34$ \\
\hline $\begin{array}{r}\text { Length category } \\
<100 \mathrm{~cm} \\
\end{array}$ & $21(70.0 \%)$ & & \\
\hline$>100 \mathrm{~cm}$ & $9 \quad(30.0 \%)$ & & \\
\hline
\end{tabular}

Table (1) showed the demographic data of the patients enrolled in the current study.

Table (2): Echocardiographic diagnosis of cardiac lesions and type of cardiac catheterization

\begin{tabular}{|l|c|c|c|}
\hline \multicolumn{2}{|c|}{} & $\mathbf{N o = 3 0}$ & $\mathbf{\%}$ \\
\hline \multirow{4}{*}{$\begin{array}{l}\text { Echocardiographic } \\
\text { diagnosis }\end{array}$} & Muscular VSD & 6 & $20.0 \%$ \\
\cline { 2 - 4 } & Secondum ASD & 12 & $40.0 \%$ \\
\cline { 2 - 4 } & Fallot tetralogy & 4 & $13.3 \%$ \\
\cline { 2 - 4 } & Small PDA & 4 & $13.3 \%$ \\
\cline { 2 - 4 } & Moderate PDA & 4 & $13.3 \%$ \\
\hline \multirow{2}{*}{$\begin{array}{l}\text { Cardiac } \\
\text { catheterization }\end{array}$} & Diagnostic & 5 & $16.7 \%$ \\
\cline { 2 - 4 } & Therapeutic & 25 & $83.3 \%$ \\
\hline
\end{tabular}


Table (2) showed that according to echocardiographic diagnosis, majority of cases had secondum ASD with percentage $40 \%$, whereas percentage of cases with muscular VSD was $20 \%$ and percentage of fallot tetralogy cases, small PDA cases, and moderate PDA cases was $13.3 \%$. Also, according to type of cardiac catheterization, percentage of therapeutic cardiac catheterization was $83.3 \%$ whereas diagnostic cardiac catheterization was $16.7 \%$.

Table (3): Echocardiographic data of the patients

\begin{tabular}{|c|c|}
\hline & No. $=30$ \\
\hline \multicolumn{2}{|c|}{ Estimated pulmonary artery pressure $(\mathrm{mm} \mathrm{Hg})$} \\
\hline Mean \pm SD & $24.07 \pm 11.69$ \\
\hline \multicolumn{2}{|c|}{ Pulmonary artery pressure category $(\%)$} \\
\hline$<24 \mathrm{mmHg}$ & $19(63.3 \%)$ \\
\hline$>24 \mathrm{mmHg}$ & $11(36.7 \%)$ \\
\hline \multicolumn{2}{|l|}{ F.S (\%) } \\
\hline Mean \pm SD & $39.34 \pm 5.71$ \\
\hline \multicolumn{2}{|l|}{ FS category } \\
\hline$<40 \%$ & $17(56.7 \%)$ \\
\hline$>40 \%$ & $13(43.3 \%)$ \\
\hline \multicolumn{2}{|l|}{ E.F (\%) } \\
\hline Mean \pm SD & $65.68 \pm 5.08$ \\
\hline \multicolumn{2}{|l|}{ EF category } \\
\hline$<65 \%$ & $11(36.7 \%)$ \\
\hline$>65 \%$ & $1963.3 \%)$ \\
\hline
\end{tabular}

Table (3) showed that the mean estimated pulmonary artery pressure was $24.07 \pm 11.69 \mathrm{~mm} \mathrm{Hg}$, the mean F.S was $39.34 \pm 5.71$ and the mean E.F was $65.68 \pm 5.08 \%$.

Table (4): Mean hs-cTnI levels at baseline, immediately after and 12 hours after cardiac catheterization

\begin{tabular}{|c|c|c|c|}
\hline & Mean \pm SD & $\mathbf{t}$ & P-value \\
\hline hs-cTnI before $\quad(\mathrm{ng} / \mathrm{ml})$ & $0.008 \pm 0.004$ & - & - \\
\hline hs-cTnI immediately after (ng/ml) & $0.009 \pm 0.004$ & -9.709 & $<0.001^{*}$ \\
\hline hs-cTnI after12hrs $\quad(\mathrm{ng} / \mathrm{ml})$ & $0.013 \pm 0.004$ & -10.588 & $<0.001^{*}$ \\
\hline
\end{tabular}

P-value <0.05: Significant; P-value< 0.001: highly significant; t: Paired t- test*: P-value hs-cTnI before Vs hs-cTnI immediately after.**: P-value hs-cTnI before Vs hs-cTnI after12 hrs.

Table (4) showed that the mean serum hs-cTnI level before pediatric cardiac catheterization was $(0.008$ $\pm 0.004 \mathrm{ng} / \mathrm{ml})$, whereas the mean serum hs-cTnI level immediately after pediatric cardiac catheterization was $(0.009$ $\pm 0.004 \mathrm{ng} / \mathrm{ml})$ and after 12 hours was $(0.013 \pm 0.004 \mathrm{ng} / \mathrm{ml})$, with highly significant increase of serum hs-cTnI level immediately after cardiac catheterization in comparison to before it ( $\mathrm{p}$-value $<0.001$ ), also highly significant increase of serum hs-c TnI level after 12 hours from cardiac catheterization in comparison to before it ( $\mathrm{p}$-value $<0.001$ ). 
Table (5): Comparison of serum hs-cTnI levels at baseline, immediately after and 12 hours after cardiac catheterization according to demographic data, echocardiographic data and type of cardiac catheterization

\begin{tabular}{|c|c|c|c|c|c|c|c|}
\hline & \multirow{2}{*}{\begin{tabular}{|c|}
$\begin{array}{c}\text { hs-cTnI before } \\
(\mathrm{ng} / \mathrm{ml})\end{array}$ \\
Mean \pm SD \\
\end{tabular}} & \multirow{2}{*}{$\begin{array}{c}\text { hs-cTnI after } \\
(\mathrm{ng} / \mathrm{ml})\end{array}$} & \multirow{2}{*}{$\begin{array}{c}\begin{array}{c}\text { hs-cTnI } \\
\text { after12hrs } \\
(\mathrm{ng} / \mathrm{ml})\end{array} \\
\text { Mean } \pm \text { SD }\end{array}$} & \multirow[t]{2}{*}{ P1 } & \multirow[t]{2}{*}{$\mathbf{P 2}$} & \multirow[t]{2}{*}{ P3 } \\
\hline & & & & & & & \\
\hline \multirow[t]{2}{*}{ Age } & $<4$ years & $0.009 \pm 0.004$ & $0.010 \pm 0.004$ & $0.014 \pm 0.003$ & $<0.001$ & $<0.001$ & \\
\hline & $>4$ years & $0.007 \pm 0.004$ & $0.008 \pm 0.004$ & $0.012 \pm 0.004$ & 0.002 & $<0.001$ & $>0.05$ \\
\hline \multirow[t]{2}{*}{ Sex } & \begin{tabular}{|l} 
Females \\
\end{tabular} & $0.009 \pm 0.004$ & $0.010 \pm 0.004$ & $0.013 \pm 0.004$ & $<0.001$ & $<0.001$ & \\
\hline & Males & $0.008 \pm 0.004$ & $0.009 \pm 0.004$ & $0.013 \pm 0.004$ & $<0.001$ & $<0.001$ & $>0.05$ \\
\hline \multirow[t]{2}{*}{ Weight } & $5 \mathrm{~kg}$ & $0.009 \pm 0.004$ & $0.010 \pm 0.004$ & $0.014 \pm 0.003$ & $<0.001$ & $<0.001$ & \\
\hline & $5 \mathrm{~kg}$ & $0.007 \pm 0.004$ & $0.009 \pm 0.004$ & $0.012 \pm 0.004$ & 0.003 & $<0.001$ & $>0.05$ \\
\hline \multirow[t]{5}{*}{ Echo diagnosis } & \begin{tabular}{|l|} 
Muscular- \\
VSD \\
\end{tabular} & $0.008 \pm 0.005$ & $0.009 \pm 0.004$ & $0.012 \pm 0.004$ & 0.013 & 0.004 & \\
\hline & $\begin{array}{l}\text { Secondum- } \\
\text { ASD }\end{array}$ & $0.008 \pm 0.004$ & $0.009 \pm 0.004$ & $0.014 \pm 0.004$ & $<0.001$ & $<0.001$ & \\
\hline & Fallot & $0.008 \pm 0.004$ & $0.009 \pm 0.004$ & $0.012 \pm 0.005$ & 0.092 & 0.092 & $>0.05$ \\
\hline & Small PDA & $0.008 \pm 0.004$ & $0.009 \pm 0.004$ & $0.012 \pm 0.003$ & $<0.001$ & 0.110 & \\
\hline & $\begin{array}{l}\text { Moderate } \\
\text { PDA }\end{array}$ & $0.011 \pm 0.004$ & $0.011 \pm 0.004$ & $0.015 \pm 0.002$ & 0.133 & 0.053 & \\
\hline \multirow[t]{2}{*}{ Catheterization } & Diagnostic & $0.008 \pm 0.003$ & $0.009 \pm 0.003$ & $0.012 \pm 0.004$ & 0.099 & 0.099 & \\
\hline & Therapeutic & $0.009 \pm 0.004$ & $0.010 \pm 0.004$ & $0.013 \pm 0.004$ & $<0.001$ & $<0.001$ & \\
\hline \multirow{2}{*}{$\begin{array}{c}\text { Pulmonary } \\
\text { artery pressure }\end{array}$} & $<24 \mathrm{mmHg}$ & $0.008 \pm 0.004$ & $0.009 \pm 0.004$ & $0.013 \pm 0.004$ & $<0.001$ & $<0.001$ & \\
\hline & $>24 \mathrm{mmHg}$ & $0.009 \pm 0.004$ & $0.010 \pm 0.004$ & $0.014 \pm 0.003$ & $<0.001$ & $<0.001$ & $>0.05$ \\
\hline \multirow[t]{2}{*}{ FS } & $<40 \%$ & $0.009 \pm 0.004$ & $0.010 \pm 0.004$ & $0.013 \pm 0.004$ & $<0.001$ & $<0.001$ & \\
\hline & $>40 \%$ & $0.008 \pm 0.003$ & $0.009 \pm 0.003$ & $0.013 \pm 0.004$ & $<0.001$ & $<0.001$ & $>0.05$ \\
\hline \multirow[t]{2}{*}{$\mathbf{E F}$} & $<65 \%$ & $0.009 \pm 0.005$ & $0.010 \pm 0.004$ & $0.013 \pm 0.004$ & 0.001 & $<0.001$ & \\
\hline & $>65 \%$ & $0.008 \pm 0.003$ & $0.009 \pm 0.004$ & $0.013 \pm 0.004$ & $<0.001$ & $<0.001$ & $>0.05$ \\
\hline
\end{tabular}

P1: hs-cTnI before vs hs-cTnI immediately after pediatric cardiac catheterization.P2: hs-cTnI before vs hs-cTnI after 12 hours of pediatric cardiac catheterization.P3: comparison of hs-cTnI between different demographic and echocardiographic data.

Table (5) showed that there was highly significant increase of serum hs-cTnI levels immediately after and 12 hours after therapeutic pediatric cardiac catheterization $(\mathrm{p}<0.001)$, whereas in diagnostic pediatric cardiac catheterization there was no significant differences of serum hs-cTnI levels ( $p>0.05)$. also, there was no significant differences of serum hs-cTnI levels according to age, sex, weight, pulmonary artery pressure, EF \% or FS \% (P >0.05). Also, there was no significant differences of serum hs-cTnI regarding type of cardiac lesions $(\mathrm{p}>0.05)$. 
Ahmed Hegazy et al.
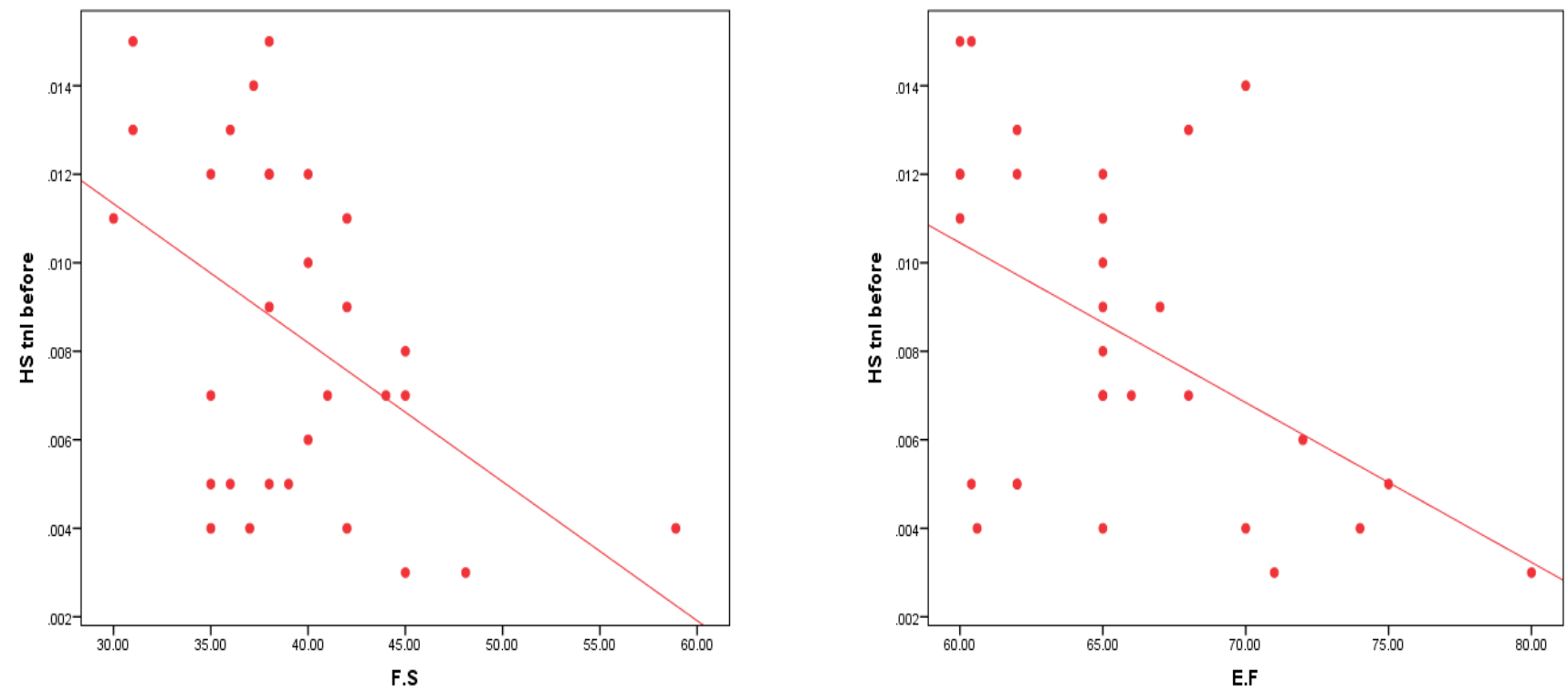

Figure (1): Correlation between serum hs-cTnI levels at Figure (2): Correlation between serum hs-cTnI levels at baseline before cardiac catheterization and FS\%.

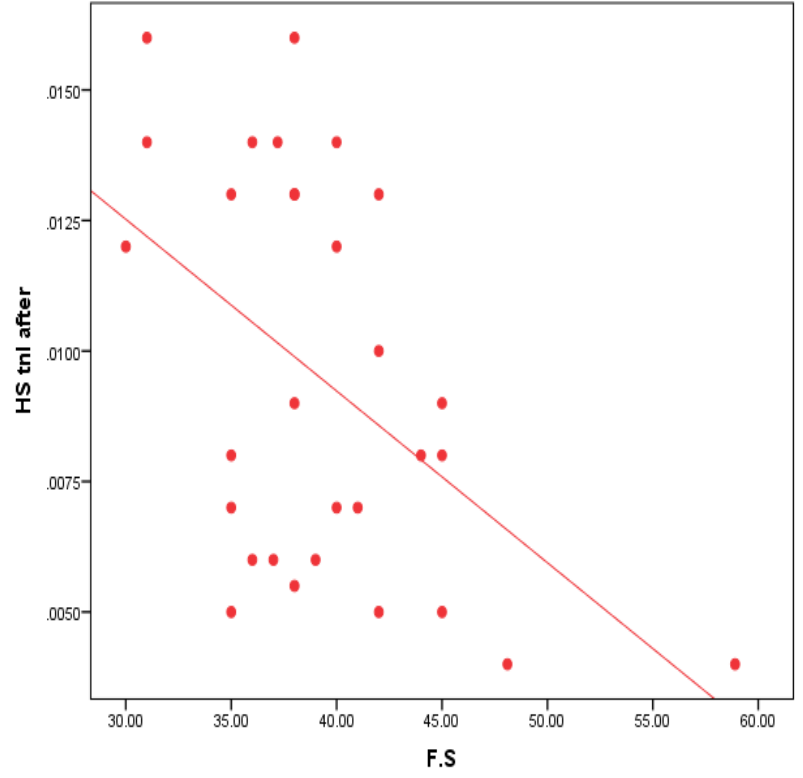

Figure (3): Correlation between serum hs-cTnI levels immediately after cardiac catheterization and FS \%. baseline before cardiac catheterization and $\mathrm{EF} \%$.

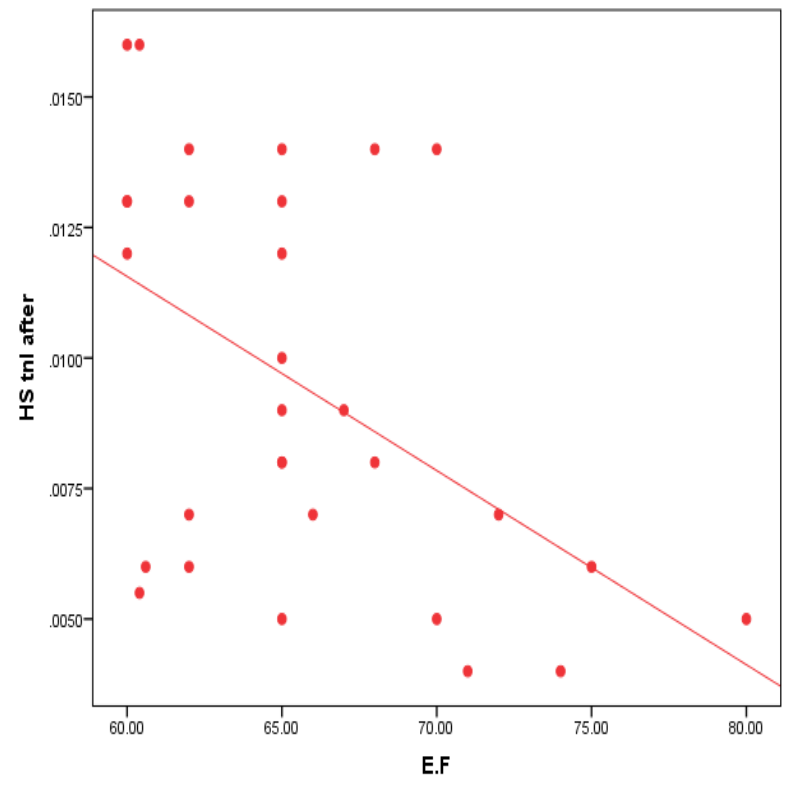

Figure (4): Correlation between serum hs-cTnI levels immediately after cardiac catheterization and $\mathrm{EF} \%$. 


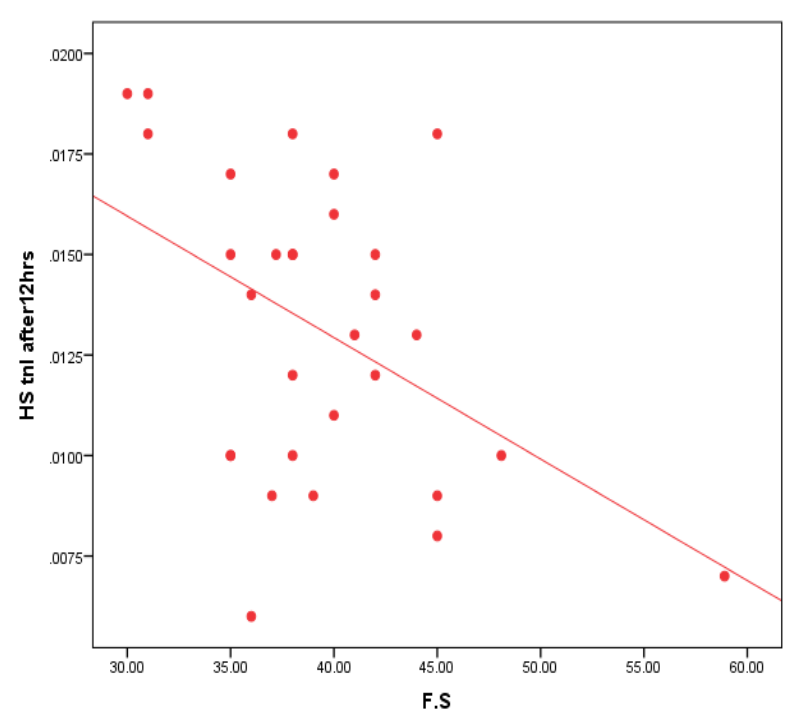

Figure (5): Correlation between mean serum hs-cTnI levels 12 hours after cardiac catheterization and FS \%.

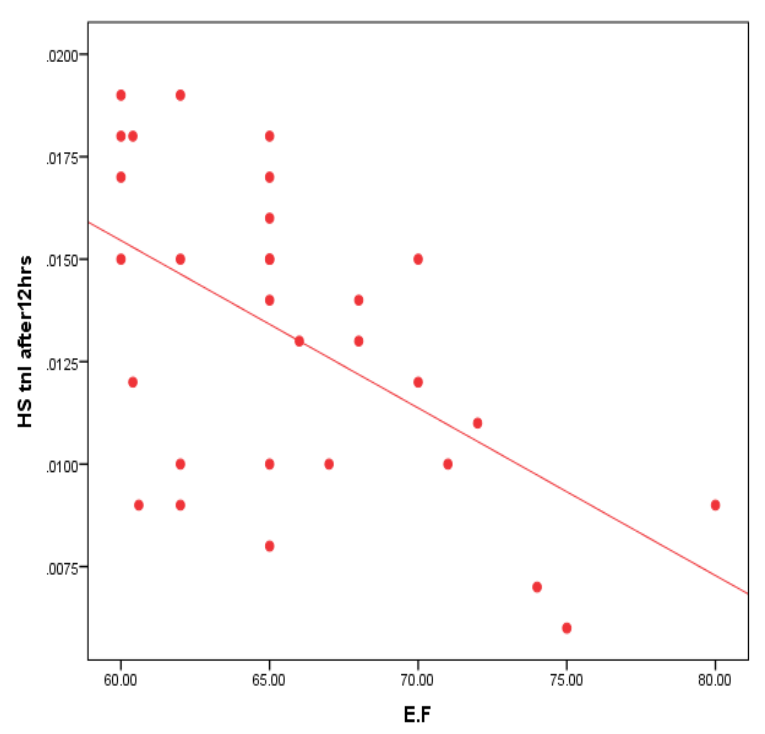

Figure (6): Correlation between serum hs-cTnI levels 12 hours after cardiac catheterization and EF \%.

Figures (1-6) showed that there was significant negative correlation between mean serum hs-cTnI levels before, immediately after and 12 hours after pediatric cardiac catheterization with FS\%, and also there was significant negative correlation between mean serum hs-cTnI levels before, immediately after and 12 hours after pediatric cardiac catheterization with $\mathrm{EF} \%(\mathrm{P}<0.05)$.

\section{DISCUSSION}

Cardiac troponin $\mathrm{I}$ is a sensitive and specific marker for detecting myocardial damage. This biomarker is normally not found in the circulation. Injured cardiac muscles release troponin from the contractile proteins into the blood stream. Elevated serum cardiac troponin I levels have been reported in $5 \%$ to $40 \%$ of patients undergoing percutaneous coronary intervention ${ }^{(\mathbf{( b )}}$.

High-sensitivity cardiac troponin (hs-cTn) assays can measure approximately 10 times lower concentrations with high precision (coefficient of variation, $<10 \%$ at the $99^{\text {th }}$ percentile of the upper reference limit) than conventional assays, and can measure cTn concentrations in at least $50 \%$ of a reference population. Because high-sensitivity cTn assays can detect smaller amounts of myocardial injury within a shorter time after the onset of symptoms, they improve the early diagnosis, particularly early rule out of acute myocardial infarction (AMI). Improvements in analytical sensitivity have also increased the number of cardiac troponin (cTn) elevations in various acute and chronic conditions with cardiac involvement other than AMI $^{(7)}$.

Feldman et al $^{\left({ }^{(8)}\right.}$ indicated that cardiac troponin I or cardiac troponin $\mathrm{T}$ elevation after elective percutaneous coronary intervention is indicative of an increase in long-term all-cause mortality as well as the composite adverse events of all-cause mortality of acute coronary syndromes. The safety of diagnostic cardiac catheterization has been demonstrated by West $\boldsymbol{e t}$ al. ${ }^{\left({ }^{(9)}\right.}$ who showed very rare clinical complications following the procedure.

In a pediatric population, Kannankeril et al. ${ }^{(10)}$ documented elevations in serum cardiac troponin I levels occurring in $79 \%$ of cases immediately after the procedure, and in $86 \%$ of them six hours post-diagnostic cardiac catheterization. However, these elevations in serum cardiac troponin I have not yet been shown to be predictive of future adverse cardiac events. The authors highlighted the need for a cut-off value for the initiation of close monitoring for possible procedure-related complications where myocardial injury is suspected.

Sharma and Jackson (11) showed substantial elevations in serum cardiac troponin I following diagnostic cardiac catheterization in children. Peng et al. (12) also determined the occurrence of myocardial injury due to diagnostic cardiac catheterization procedure in adults evaluating the elevation in serum cardiac troponin I levels. A prospective cohort study was carried out among patients who underwent diagnostic cardiac catheterization in a tertiary hospital. Blood samples were collected at baseline and at 6- and 12- hours of the procedure to measure any changes in cardiac troponin I levels. Average cardiac troponin I levels at 6- and 12hours following diagnostic cardiac catheterization did not differ according to patients' demographic or clinical 
characteristics. There were no significant changes in cardiac troponin I levels before or at 6- and 12-hours after diagnostic cardiac catheterization. Therefore, they suggested that diagnostic cardiac catheterization does not appear to be associated with substantial subclinical myocardial injury.

In the present study, there was significant elevation of serum highly sensitive cardiac troponin I (hs-cTnI) immediately after therapeutic pediatric cardiac catheterization, also there was significant elevation of serum hs-cTnI level 12 hours following cardiac catheterization. These findings are in agreement with the study of Kannankeril et al. ${ }^{(10)}$ in which 14 pediatric patients were included. Levels of cardiac troponin I was elevated in the serum from 11 of $14(79 \%)$ cases immediately after the procedure, and in 12of 14 (86\%) six hours after therapeutic cardiac catheterization. These findings are also near to findings of Pahl et al. ${ }^{(13)}$ who found that most pediatric interventional catheterization procedures were associated with myocardial injury, as evidenced by elevation of cTnI, with radiofrequency catheter ablation (RFA) causing higher levels than other interventions. Conversely, most diagnostic procedures were associated with no detectable myocardial injury. In their study, seventy-three pediatric catheterizations were evaluated. Diagnostic procedures and interventions not expected to cause myocardial injury were assigned to group I, whereas interventional procedures expected to be associated with cardiac injury were assigned to group II. Procedures in group II were associated with significantly higher cTnI levels than group I. When compared with Peng et al. ${ }^{(12)}$ study about diagnostic cardiac catheterization, pediatric patients seem to be at higher risk for myocardial injury from interventional cardiac catheterization.

In the current study, the mean serum hs-cTnI level immediately after or 12 hours after diagnostic cardiac catheterization did not differ significantly. This is in agreement with Kannankeril et al. ${ }^{(10)}$ and Peng et al. ${ }^{(12)}$ who reported that most diagnostic procedures were associated with no detectable myocardial injury. On the other hand, a study done by Baysal et al. ${ }^{(14)}$ in which the study group consisted of 30 pediatric patients (17 males, 13 females), undergoing diagnostic cardiac catheterization, 18 patients $(60 \%)$ had serum cardiac TnI levels above $0.6 \mathrm{ng} / \mathrm{ml}$, and 16 patients $(53 \%)$ had serum CK- MB levels above 35 U/L. The cardiac TnI and CKMB levels taken after the procedures were significantly higher than the values taken before the procedures.

Also, a study was performed by pees et al. ${ }^{(15)}$ to assess possible myocardial injury caused by interventional closure of atrial septal defects (ASDs) compared to diagnostic catheterization by measuring cardiac troponin I (cTn-I). Forty patients were enrolled;
33 ASDs were successfully closed, while in 7 cases diagnostic balloon sizing of the defect was performed only. No significant increase could be detected in patients with diagnostic balloon sizing. Following interventional closure of ASDs with Amplatzer septum/PFO occluders, increased cTn-I levels for several hours indicated some transient, reversible myocardial membrane instability due to the device.

In comparison to other biochemical markers for myocardial injury, a study was performed by Nageh $\boldsymbol{e t}$ al. ${ }^{(16)}$ on 109 patients to determine the most sensitive biochemical marker for the detection of cardiac myocyte damage potentially sustained during percutaneous coronary intervention (PCI) and to assess whether such a marker can be used to identify patients at increased risk of poor subsequent clinical outcome. CTnI had the highest detection rate for myocardial damage, with 58 cTnI-positive patients, 38 cTnT-positive patients and 28 CK-MB-positive patients in the 24 hours following PCI.

The present study showed that there was no significant differences of serum hs-cTnI levels according to age, sex, weight or pulmonary artery pressure $(\mathrm{P}$ $>0.05$ ). Also, there was no significant differences of serum hs-cTnI regarding type of cardiac lesions. This is not in agreement with Kannankeril et al. ${ }^{(1)}$ who showed that cTnI was inversely correlated with age and weight; younger and smaller patients tended to have higher levels of cTnI. The authors suggested that immature myocardium is more vulnerable to injury from a variety of causes, including surgery and cardiac catheterization and the patient size and age are important risk factors for myocardial injury after invasive cardiac procedures.

The present study also showed that there was significant negative correlation between serum hs-cTnI levels before, immediately after and 12 hours after pediatric cardiac catheterization with $\mathrm{FS} \%$, and also there was significant negative correlation between serum hs-cTnI levels before, immediately after and 12 hours after pediatric cardiac catheterization with $\mathrm{EF} \%$ $(\mathrm{P}<0.05)$, denoting inverse correlation between this cardiac biomarker and systolic cardiac function, which was mentioned in most researches. The potential clinical benefit of this study is in high lighting the need for a cut-off value for the initiation of close monitoring for possible procedurerelated complications where myocardial injury is suspected. In such cases, the clinical relevance of high levels of serum cardiac troponin I found in the patients may be difficult to interpret unless a good knowledge of the expected procedure-related elevation after an uneventful procedure is known. Therefore, establishing a reference range of "expected"/normal elevation would allow levels of serum cardiac troponin I to be used as a 
valid diagnostic tool to detect "unexpected/sub-clinical" myocardial injury following diagnostic/therapeutic cardiac catheterization.

In conclusion; serum highly sensitive cardiac troponin I (hs-cTnI) was significantly increased immediately after pediatric cardiac catheterization, with more significant increase after 12 hours, suggesting myocardial injury in these patients. Also, elevation of this diagnostic biomarker of myocardial injury was more after therapeutic than diagnostic pediatric cardiac catheterization.

\section{REFERENCES}

1. Feltes T, Bacha E, Beekman III et al. (2011): Indications for cardiac catheterization and intervention in pediatric cardiac disease: a scientific statement from the American Heart Association. Circulation, 123(22): 2607-2652.

2. Wu A, Feng Y, Moore R et al. (1998): Characterization of cardiac troponin subunit release into serum after acute myocardial infarction and comparison of assays for troponin T and I. Clinical chemistry, 44(6): 1198-1208.

3. Sherwood M and Kristin Newby L (2014): Highsensitivity troponin assays: evidence, indications, and reasonable use. Journal of the American Heart Association, 3(1): e000403.

4. Moustafa G, Kolokythas A, Charitakis K et al. (2016): Diagnostic Cardiac Catheterization in the Pediatric Population. Current cardiology reviews, 12(2), 155-162. doi:10.2174/1573403X12666160301120955.

5. Lindahl B, Venge $P$ and James $S$ (2010): The new highsensitivity cardiac troponin $\mathrm{T}$ assay improves risk assessment in acute coronary syndromes. American Heart Journal, 160(2): 224-229.

6. Gaze $D$ and Collinson $P$ (2005): Cardiac troponins as biomarkers of drug-and toxin-induced cardiac toxicity and cardio protection. Expert opinion on drug metabolism \& toxicology, 1(4): 715-725.
7. Thygesen K, Alpert J, Jaffe A et al. (2012): Third universal definition of myocardial infarction. Circulation, 126(16): 2020-2035.

8. Feldman D, Kim L, Rene A et al. (2011):Prognostic value of cardiac troponin- I or troponin- $\mathrm{T}$ elevation following non-emergent percutaneous coronary intervention: A meta- analysis. Catheterization and Cardiovascular Interventions, 77(7): 1020-1030.

9. West R, Ellis G and Brooks N (2006): Complications of diagnostic cardiac catheterization: results from a confidential inquiry into cardiac catheter complications. Heart, 92(6): 810-814.

10. Kannankeril P, Pahl E and Wax D (2002): Usefulness of troponin I as a marker of myocardial injury after pediatric cardiac catheterization. The American journal of cardiology, 90(10): 1128-1132.

11.Sharma S, Jackson PG and Makan J (2004):Cardiac troponins Journal of Clinical Pathology, (57):1025-1026.

12. Peng J, Jiang $Q$, Cheng $Z$ et al.(2015): Analysis Of Troponin I To Determine Myocardial Injury Following Diagnostic Cardiac Catheterization. Biomedical Research , 26:4.

13. Pahl E, Naftel D, Kuhn M et al. (2005): The impact and outcome of transplant coronary artery disease in a pediatric population: a 9-year multi-institutional study. The Journal of Heart Lung Transplantation, 24(6): 645651.

14. Baysal T, Oran B, Başpinar O et al. (2005): Cardiac troponin I elevation in pediatric cardiac catheterization. Anadolukardiyolojidergisi: $\mathrm{AKD}=$ the Anatolian journal of cardiology, 5(2): 112-115.

15. Pees C, Haas $\mathbf{N}$, von der Beek $\mathbf{J}$ et al. (2003):Cardiac troponin I is increased after interventional closure of atrial septal defects. Catheterization and cardiovascular interventions, 58(1): 124-129.

16. Nageh $T$, Sherwood R, Harris $B$ et al. (2003): Cardiac troponin $\mathrm{T}$ and $\mathrm{I}$ and creatine kinase-MB as markers of myocardial injury and predictors of outcome following percutaneous coronary intervention. International journal of cardiology, 92(2-3): 285-293. 